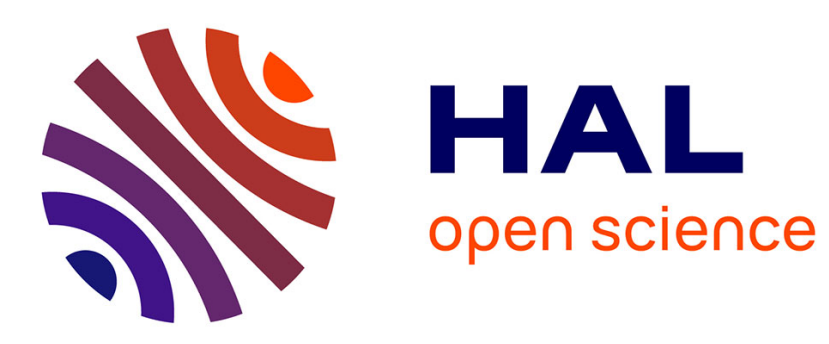

\title{
Dynamic analysis and control of an antagonistically actuated tensegrity mechanism
}

Anders van Riesen, Matthieu Furet, Christine Chevallereau, Philippe Wenger

\section{To cite this version:}

Anders van Riesen, Matthieu Furet, Christine Chevallereau, Philippe Wenger. Dynamic analysis and control of an antagonistically actuated tensegrity mechanism. 22nd CISM IFToMM Symposium on Robot Design, Dynamics and Control (ROMANSY'2018), Jun 2018, Rennes, France. pp.481-490, 10.1007/978-3-319-78963-7_60. hal-01989963

\section{HAL Id: hal-01989963 https://hal.science/hal-01989963}

Submitted on 22 Jan 2019

HAL is a multi-disciplinary open access archive for the deposit and dissemination of scientific research documents, whether they are published or not. The documents may come from teaching and research institutions in France or abroad, or from public or private research centers.
L'archive ouverte pluridisciplinaire HAL, est destinée au dépôt et à la diffusion de documents scientifiques de niveau recherche, publiés ou non, émanant des établissements d'enseignement et de recherche français ou étrangers, des laboratoires publics ou privés. 


\title{
Dynamic analysis and control of an antagonistically actuated tensegrity mechanism
}

\author{
Anders van Riesen ${ }^{1,2}$, Matthieu Furet ${ }^{1}$, Christine Chevallereau ${ }^{1}$, and Philippe \\ Wenger $^{1}$ \\ 1 Laboratoire des Sciences du Numérique de Nantes (LS2N), CNRS, Ecole centrale \\ de Nantes, 44321 Nantes, France \\ 2 University of Twente, 7500 AE Enschede, The Netherlands
}

\begin{abstract}
This paper analyses the dynamics of an antagonistically actuated tensegrity mechanism. The mechanism is subject to gravity effects, which produce both stable and unstable equilibrium configurations. The workspace is shown to be not necessarily connected and its size depends on both the geometric, spring and actuator parameters of the mechanism. The antagonistic actuation forces, which are bounded, enable controlling both the stiffness and the position within certain limits. A computed torque control law is applied and simulations show interesting behaviors of the mechanism when the desired motion makes the mechanism jump between two connected components of the workspace.
\end{abstract}

Keywords: Tensegrity, Mechanism, Dynamics, Stiffness, Control

\section{Introduction}

A tensegrity structure is an assembly of compressive elements (bars) and tensile elements (cable, springs) held together in equilibrium [1]. A tensegrity mechanism is obtained when one or several elements are actuated. They exhibit interesting properties such as low inertia, natural compliance and deployability [2], [3]. This work falls within the context of the AVINECK project involving biologists and roboticists with the main objective to model and design bird necks. Accordingly, a class of planar tensegrity manipulators made of a series assembly of several Snelson's X-shape mechanisms [4] has been chosen as a suitable candidate for a preliminary planar model of a bird neck, see figure 1. Snelson's X-shape mechanisms have been studied by a number of researchers, either as a single mechanism [2],[3],[5] or assembled in series [6],[7],[8]. Here, a single mechanism is actuated with two lateral tendons threaded through the spring attachment points like in [8]. This mechanism has only two lateral springs and the upper link is rigid, contrary to the ones analyzed in [2],[3],[5],[6],[7]. Moreover, the mechanism is supposed to operate in a vertical plane and is thus subject to gravity, unlike in [8]. The mechanism is antagonistically actuated, which allows controlling its stiffness in addition to its motion [9]. This paper focuses on the dynamic analysis of the mechanism [10], the impact of the spring stiffness on the range of stable positions, and the control of such a system. It is found that the 
system has stable and unstable equilibrium positions, a property that was not observed in the absence of gravity. It is shown that the ratio between the bar mass and the spring stiffness has a great influence on the size of the workspace. Moreover, the workspace may not be continuous. To actuate the system, a control scheme is proposed, which takes into account the admissible tension in the cables and manages priority between the position and the stiffness control.

\section{Mechanism description}

A single module of the bird neck model consists of a four-bar mechanism with crossed links and two pre-tensioned springs. The links are rigid, homogeneous and linear and are not subject to buckling. They are connected by A, B, C and $\mathrm{D}$, assumed to be perfect revolute joints with no friction and damping, see Fig. 1. The mechanism is fixed to the ground by A and B. The two springs connect $\mathrm{A}$ and $\mathrm{D}$, and $\mathrm{B}$ and $\mathrm{C}$, and are considered massless and of zero free length. The mechanism data are given in Tab. 1. The two crossed links have the same length $L$ and the base and top links have the same length $b$.

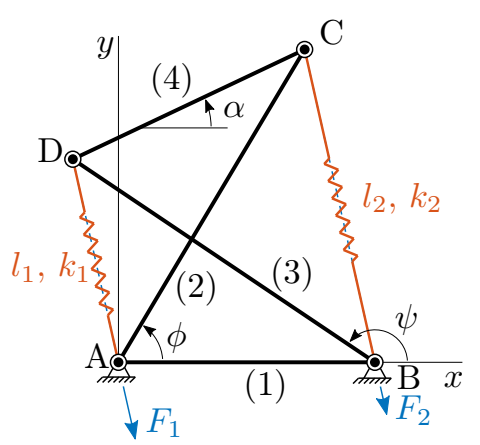

Fig. 1: Single module.

\begin{tabular}{cccc}
\hline & Length & Mass & Stiffness \\
\hline \hline Link 1 & $b$ & $m_{1}$ & - \\
Link 2 & $L$ & $m_{2}$ & - \\
Link 3 & $L$ & $m_{3}$ & - \\
Link 4 & $b$ & $m_{4}$ & - \\
Spring 1 & $l_{1}$ & - & $k_{1}$ \\
Spring 2 & $l_{2}$ & - & $k_{2}$ \\
\hline
\end{tabular}

Table 1: Mechanism data.

The mechanism is actuated by tendons connected at hinges $\mathrm{D}$ and $\mathrm{C}$ and run through the springs. The tendons are considered to have an infinite stiffness, hence input forces $F_{1}$ and $F_{2}$ are considered to act directly on D and C, respectively. Forces $F_{1}$ and $F_{2}$ are bounded by $F_{\max }$ and $F_{\min }$ and since tendons cannot push, $F_{\min }$ is positive. The rotation range of the mechanism is $-\pi<\alpha<\pi$, i.e. the mechanism cannot reach the flat singular configurations.

\section{$3 \quad$ Kinematic analysis}

Movable links 2, 3 and 4 are considered for the kinematic and dynamic analysis. Their orientation is defined by their orientation angle $\phi, \psi$ and $\alpha$, respectively (Fig. 1). These are chosen as the generalized coordinates. The mechanism has one degree of freedom and $\alpha$ is chosen to specify the mechanism configuration. Considering Fig. 1 again, the coordinate frame is defined such that the origin 
coincides with hinge $\mathrm{A}$ and the $\mathrm{x}$-axis is aligned with link 1 . Decomposition of the loop-closure constraint equation (written in $\mathrm{A}$ ) in $\mathrm{x}$ - and $\mathrm{y}$-direction yields:

$$
\begin{array}{r}
b+L \cos (\psi)+b \cos (\alpha)-L \cos (\phi)=0 \\
L \sin (\psi)+b \sin (\alpha)-L \sin (\phi)=0
\end{array}
$$

Solving Eq. 1 by expressing the dependent coordinate as a function of the independent coordinates yields the constraint equations

$$
\begin{aligned}
& \phi(\alpha)=2 \arctan \left[\frac{2 b L \sin (\alpha)+S}{\left(2 b^{2}+2 b L\right)[\cos (\alpha)+1]}\right] \\
& \psi(\alpha)=2 \arctan \left[\frac{-2 b L \sin (\alpha)-S}{\left(2 b^{2}-2 b L\right)[\cos (\alpha)+1]}\right]
\end{aligned}
$$

with $S=\sqrt{(-2 b L \sin (\alpha))^{2}+(-2 b L[\cos (\alpha)+1])^{2}-\left(2 b^{2}[\cos (\alpha)+1]\right)^{2}}$.

Note that the signs of Eqs. (2) are chosen in accordance with the allowable range of $\alpha,-\pi<\alpha<\pi$. Equations (1) make it possible to calculate the first and second derivatives of $\phi$ and $\psi$ with respect to $\alpha$, which are useful for the dynamic model presented in Sec. 4. The expressions for the spring lengths, useful for the next section, are expressed in the generalized coordinates using the cosine rule:

$$
l_{1}=\sqrt{b^{2}+L^{2}+2 b L \cos (\psi)} \quad(3 \mathrm{a}) \quad l_{2}=\sqrt{b^{2}+L^{2}-2 b L \cos (\phi)}
$$

\section{Dynamic analysis}

To simplify the writing, most equations in this section are expressions of both independent and dependent generalized coordinates. The dependent generalized coordinates must be replaced with their derived expression of section 3 .

The equation of motion of the mechanism is derived using the Lagrangian approach. Lagrange's equation is expressed as:

$$
\frac{d}{d t}\left(\frac{\partial T}{\partial \dot{\alpha}}\right)-\frac{\partial T}{\partial \alpha}+\frac{d V}{d \alpha}=Q
$$

where $T$ is the kinetic energy, $V$ is the potential energy and $Q$ the non-conservative generalized force acting on the mechanism. The kinetic energy, which depends on the (translational and rotational) velocities of the links, is:

$$
T=\left(\frac{1}{6} m_{2}+\frac{1}{2} m_{4}\right) L^{2} \dot{\phi}^{2}+\frac{1}{6} m_{3} L^{2} \dot{\psi}^{2}+\frac{1}{6} m_{4} b^{2} \dot{\alpha}^{2}-\frac{1}{2} m_{4} b L \dot{\phi} \dot{\alpha} \cos (\phi-\alpha)
$$

The potential energy due to the near earth gravity field and to the springs is:

$$
V=\frac{g}{2}\left(m_{2} L \sin (\phi)+\left(m_{3}+2 m_{4}\right) L \sin (\psi)+m_{4} b \sin (\alpha)\right)+\frac{1}{2} k_{1} l_{1}^{2}+\frac{1}{2} k_{2} l_{2}^{2}
$$

To obtain the generalized force $Q$, consider Fig. 2. The applied forces are decomposed in components tangential and normal to the circular paths $s_{1}$ and 
$s_{2}$ of points $\mathrm{D}$ and $\mathrm{C}$. Only the tangential components are of interest as the normal components do not influence the motion. They are expressed as:

$$
F_{1 t}=F_{1} \sin \left(\beta_{1}\right)=F_{1} \frac{b}{l_{1}} \sin (\psi) \quad F_{2 t}=F_{2} \sin \left(\beta_{2}\right)=F_{2} \frac{b}{l_{2}} \sin (\phi)
$$

The virtual work performed by the forces is

$$
\delta W_{e x}=F_{1 t} \delta s_{1}-F_{2 t} \delta s_{2}=\left(F_{1 t} \frac{\partial s_{1}}{\partial \psi} \frac{\partial \psi}{\partial \alpha}-F_{2 t} \frac{\partial s_{2}}{\partial \phi} \frac{\partial \phi}{\partial \alpha}\right) \delta \alpha=Q \delta \alpha
$$

The derivatives of $s_{1}$ and $s_{2}$ are $\frac{\partial s_{1}}{\partial \psi}=\frac{\partial s_{2}}{\partial \phi}=L$. With substitution of these terms and Eq. (7) in the term in front of $\delta \alpha$ of Eq. (8), the generalized force is:

$$
Q=F_{1} \frac{b L \sin (\psi)}{l_{1}(\psi)} \frac{\partial \psi}{\partial \alpha}-F_{2} \frac{b L \sin (\phi)}{l_{2}(\phi)} \frac{\partial \phi}{\partial \alpha}
$$

Substitution of Eqs. (5), (6) and (9) into Eq. (4) yields the equation of motion

$$
M(\alpha) \ddot{\alpha}+C(\alpha) \dot{\alpha}^{2}+G(\alpha)=Z_{1}(\alpha) F_{1}+Z_{2}(\alpha) F_{2}
$$

where

$$
\begin{aligned}
M(\alpha)= & \left(\frac{1}{3} m_{2} L^{2}+m_{4} L^{2}\right) \frac{\partial \phi^{2}}{\partial \alpha}+\frac{1}{3} m_{3} L^{2} \frac{\partial \psi^{2}}{\partial \alpha}+\frac{1}{3} m_{4} b^{2}-m_{4} b L \cos (\phi-\alpha) \frac{\partial \phi}{\partial \alpha} \\
C(\alpha)= & \left(\frac{1}{3} m_{2} L^{2}+m_{4} L^{2}\right) \frac{\partial \phi}{\partial \alpha} \frac{\partial^{2} \phi}{\partial \alpha^{2}}+\left(\frac{1}{3} m_{3} L^{2}\right) \frac{\partial \psi}{\partial \alpha} \frac{\partial^{2} \psi}{\partial \alpha^{2}} \\
& +\frac{1}{2} m_{4} b L \sin (\phi-\alpha)\left(\frac{\partial \phi}{d \alpha}-1\right) \frac{\partial \phi}{\partial \alpha}-\frac{1}{2} m_{4} b L \cos (\phi-\alpha) \frac{\partial^{2} \phi}{\partial \alpha^{2}} \\
G(\alpha)= & \frac{1}{2} m_{4} b g \cos (\alpha)+\left[\frac{1}{2} m_{2} g L \cos (\phi)+k_{2} b L \sin (\phi)\right] \frac{\partial \phi}{\partial \alpha} \\
& +\left[\left(\frac{1}{2} m_{3}+m_{4}\right) g L \cos (\psi)-k_{1} b L \sin (\psi)\right] \frac{\partial \psi}{\partial \alpha} \\
Z_{1}(\alpha)= & \frac{b L \sin (\psi)}{l_{1}(\psi)} \frac{\partial \psi}{\partial \alpha} \\
Z_{2}(\alpha)= & -\frac{b L \sin (\phi)}{l_{2}(\phi)} \frac{\partial \phi}{\partial \alpha}
\end{aligned}
$$




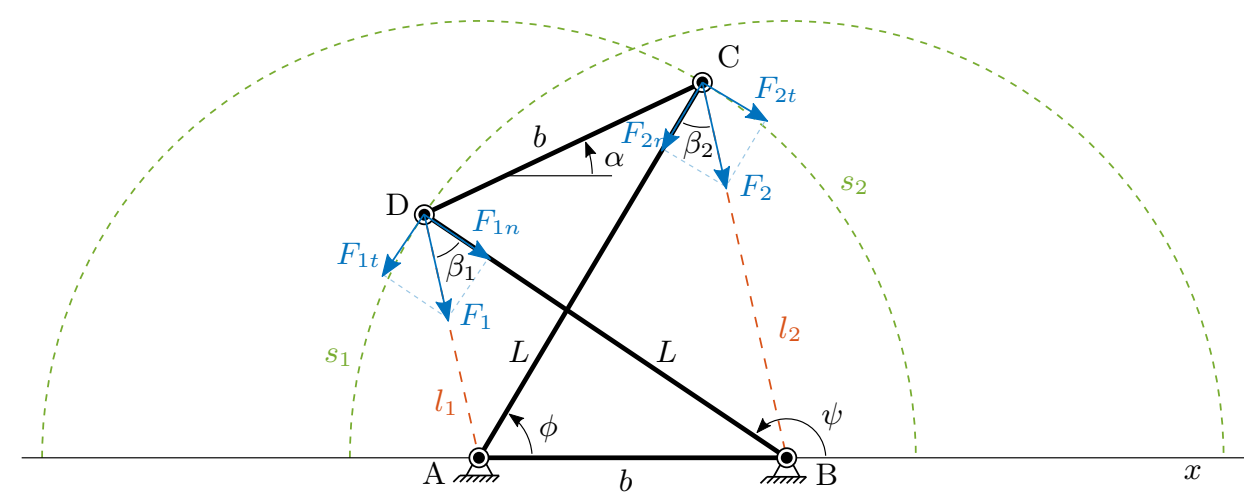

Fig. 2: Applied forces, their decompositions (blue arrows) and the circular paths (green dashed arcs) of points $D$ and $C$ of the mechanism.

\section{Workspace}

The static equilibrium condition is obtained if the time-dependent terms of Eq. (10) are omitted and $F_{1}$ and $F_{2}$ are considered as constant, which yields

$$
G(\alpha)=Z_{1}(\alpha) F_{1}+Z_{2}(\alpha) F_{2}
$$

The right-hand side of Eq. (12) is the external wrench. The wrench feasible workspace (WFW) must respect, by definition [11], [5], the kinematic constraint equations (Eq. (2)), the static equilibrium (Eq. (12)) and the limits for the external forces $F_{\max }$ and $F_{\min }$ as introduced in Sec. 2. The coefficients the right hand side of Eq. (12) defined by Eq. (11d) and (11e), have the following property:

$$
Z_{1}(\alpha)>0 \quad \text { and } \quad Z_{2}(\alpha)<0 \quad \text { for } \quad-\pi<\alpha<\pi
$$

Accordingly, it is possible to determine the limits of the external wrench based on the limits of the external forces as follows:

$$
\begin{gathered}
\Gamma_{\text {max }}(\alpha)=Z_{1}(\alpha) F_{\text {max }}+Z_{2}(\alpha) F_{\text {min }} \\
\Gamma_{\text {min }}(\alpha)=Z_{1}(\alpha) F_{\text {min }}+Z_{2}(\alpha) F_{\text {max }}
\end{gathered}
$$

The external wrench is bounded by Eq. (14). This is visualized in Fig. 3. The area enclosed by the dashed curves indicates the feasible wrench. Three instances of $G(\alpha)$ (Eq. (11c)) are present in the figure as well. Static equilibrium (Eq. (12)) is obtained for a range of $\alpha$ where $G$ is within the feasible wrench bounding curves:

$$
\Gamma_{\min }(\alpha) \leq G(\alpha) \leq \Gamma_{\max }(\alpha)
$$

The WFW is determined by the intersection of $G(\alpha)$ with $\Gamma_{\min }$ and the intersection of $G$ with $\Gamma_{\max }$. The three instances of $G$ vary in spring stiffness to illustrate their influence on the workspace. Comparing the curve $G_{k=0}$ with the 
curve $G_{k=25}$, it can be seen that increasing the spring stiffness results in a larger workspace. However, if the spring stiffness is increased, it can be seen from the $G_{k=50}$ curve that it is possible to obtain a discontinuous WFW as the curve partially exceeds the feasible wrench bounding curves. It is noteworthy that spring stiffness and mass do not have any influence on the feasible wrench.

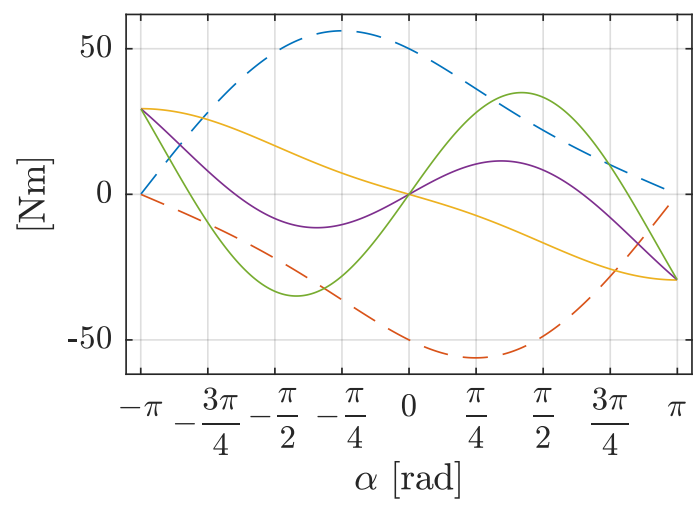

$$
\begin{aligned}
& \begin{array}{l}
---\Gamma_{\max } \\
---\Gamma_{\min } \\
G: k_{1}=k_{2}=0 \mathrm{~N} / \mathrm{m} \\
G: k_{1}=k_{2}=25 \mathrm{~N} / \mathrm{m} \\
G: k_{1}=k_{2}=50 \mathrm{~N} / \mathrm{m}
\end{array} \\
& \begin{array}{l}
F_{\text {max }}=100 \mathrm{~N}, F_{\min }=0 \mathrm{~N} \\
\rho_{l}=1 \mathrm{~kg} / \mathrm{m} \\
b=1 \mathrm{~m}, L=2 \mathrm{~m}
\end{array} \\
& \hline
\end{aligned}
$$

Fig. 3: Feasible wrench and $G(\alpha)$ for varying spring stiffness $k_{i}$.

Differentiating the static equilibrium equation (Eq. (12)) with respect to $\alpha$ gives the mechanism stiffness $K_{\alpha}$ [12]:

$$
K_{\alpha}=\frac{\partial G}{\partial \alpha}-\frac{\partial Z_{1}}{\partial \alpha} F_{1}-\frac{\partial Z_{2}}{\partial \alpha} F_{2}
$$

The stiffness is generally required to be positive to obtain a stable equilibrium. A negative stiffness means that under a small external wrench, the mechanism would leave its current configuration. For instance, this phenomenon could be used for collision avoidance in human-robot interaction. However, the naturally unstable behavior of the mechanism can be stabilized when control is applied and the WFW is not affected. Thus, a negative stiffness is allowed.

Similarly as a feasible wrench has been defined in Fig. 3 based on the limit on forces $F_{1}$ and $F_{2}$ that can be applied, a feasible mechanism stiffness is defined. From the right-hand side of Eq. (16) it is possible to determine the limits of the mechanism stiffness based on the limits of the external forces as follows:

$$
\begin{array}{ll}
K_{\alpha, \text { min }}(\alpha)=\min _{F_{\text {min }} \leq F_{i, i=1,2} \leq F_{\max }} \frac{\partial G}{\partial \alpha}-\frac{\partial Z_{1}}{\partial \alpha} F_{1}-\frac{\partial Z_{2}}{\partial \alpha} F_{2} \\
K_{\alpha, \text { max }}(\alpha)=\max _{F_{\text {min }} \leq F_{i, i=1,2} \leq F_{\max }} \frac{\partial G}{\partial \alpha}-\frac{\partial Z_{1}}{\partial \alpha} F_{1}-\frac{\partial Z_{2}}{\partial \alpha} F_{2}
\end{array}
$$

The feasible mechanism stiffness is bounded by Eq. (17), see Fig. 4. 

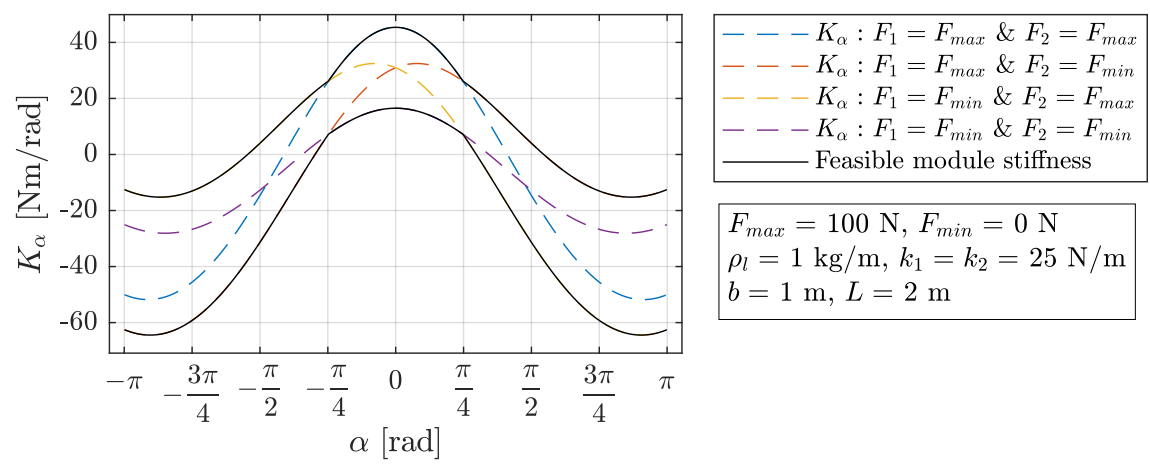

Fig. 4: Feasible stiffness.

This analysis can be used to determine the stiffness that the mechanism cannot exhibit due to its actuation. It does not imply that all the values inside the feasible area can be reached since this plot is based only on Eq. (17) and does not take into account the equation of motion (10).

\section{Control}

The control scheme of Fig. 5 is proposed. Computed torque control (CTC) is applied to track a motion $\alpha^{d}(t)$. Based on the dynamic model (10), a desired wrench $\Gamma^{d}$ is deduced:

$$
\Gamma^{d}=M(\alpha)\left(\ddot{\alpha}^{d}+K_{v}\left(\dot{\alpha}^{d}-\dot{\alpha}\right)+K_{p}\left(\alpha^{d}-\alpha\right)\right)+C(\alpha) \dot{\alpha}^{2}+G(\alpha) .
$$

The actuator forces $F_{1}, F_{2}$ able to create this desired wrench satisfy:

$$
\Gamma^{d}=Z_{1}(\alpha) F_{1}+Z_{2}(\alpha) F_{2}
$$

There is generally an infinite number of forces $F_{1}, F_{2}$ and their choice is based on a desired stiffness $K_{\alpha}^{d}$. If $\Gamma^{d}$ belongs to the feasible wrench (see Fig. 3), Eq. (19) is considered with the desired stiffness i.e.:

$$
K_{\alpha}^{d}=\frac{\partial G}{\partial \alpha}-\frac{\partial Z_{1}}{\partial \alpha} F_{1}-\frac{\partial Z_{2}}{\partial \alpha} F_{2}
$$

If $\Gamma^{d}$ does not belong to the feasible wrench, the applied wrench is saturated ( $F_{1}=F_{\max }$ and $F_{2}=F_{\min }$ or vice versa). Equations (19) and (20) allow determining $F_{1}$ and $F_{2}$. If the actuators forces exceed the force limits $F_{\min }$ or $F_{\text {max }}$, a new solution satisfying the forces limits is defined with Eq.(19) imposed and the error on Eq. (20) is minimized. Note that this control strategy prioritizes tracking over stiffness. 


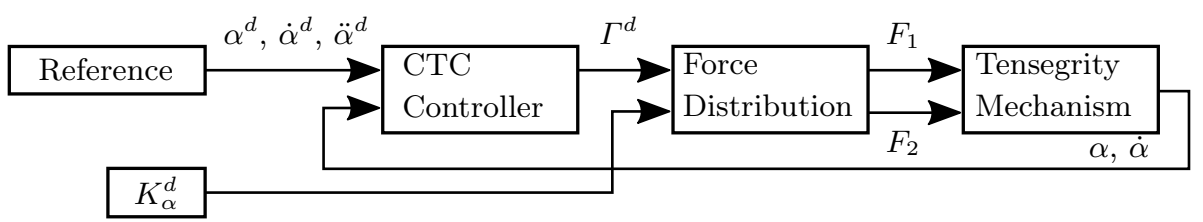

Fig. 5: Control scheme.

The result of this control strategy is now illustrated in simulation. The first simulation aims to show the behavior of the mechanism when the actuator forces are saturated due to inconsistency of the desired motion and stiffness or to the dynamics effects. The mechanism parameters used are: $F_{\max }=100 \mathrm{~N}, F_{\min }=0$ $\mathrm{N}, b=1 \mathrm{~m}, L=2 \mathrm{~m}$ and $\rho_{l}=1 \mathrm{~kg} / \mathrm{m}, k_{1}=k_{2}=25 \mathrm{~N} / \mathrm{m}$. The control gains are $K_{p}=100, K_{v}=20$.

The desired motion is composed of a set of skew-sine motions with progressive amplitude: $\pi / 4, \pi / 2$, and $3 \pi / 4$. All the desired configurations belong to the WFW as shown in Fig. 3 for $k_{1}=k_{2}=25 \mathrm{~N}$. A constant mechanism stiffness of $22 \mathrm{Nm} / \mathrm{rad}$ is desired. This stiffness belongs to the feasible stiffness only for $-\pi / 3<\alpha<\pi / 3$ as it can be seen in Fig. 4 .

Fig. 6 and 7 illustrate the desired and simulated motion, the desired and applied wrenches and the desired and simulated mechanism stiffness.

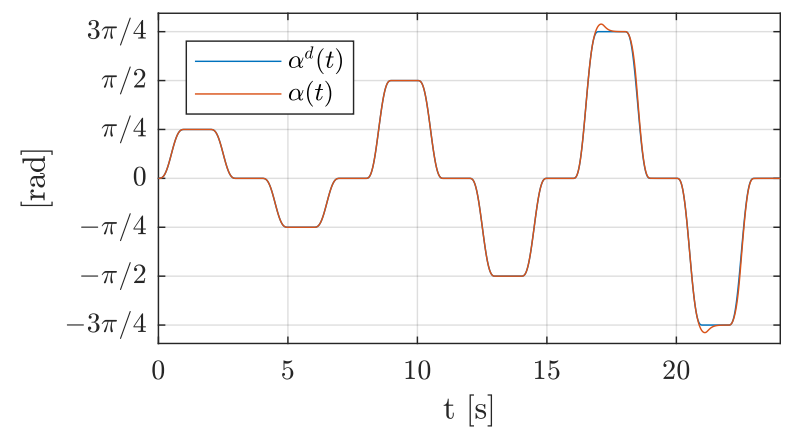

Fig. 6: Mechanism desired and simulated motions. Motion is correctly tracked for the first two sets of skew-sine motions. For the last set with the highest amplitude, tracking errors due to the saturation of actuator forces appear.

For the first set of skew-sines, of amplitude $\pi / 4$, a perfect tracking and the desired stiffness is obtained. For the second set of skew-sines, of amplitude $\pi / 2$, tracking is perfect but errors on the stiffness exist. For the third set of skewsines, of amplitude $3 \pi / 4$, the desired stiffness is not satisfied and a tracking error is observed due to dynamic effects and saturated actuator forces but with a stabilization on the desired static configuration. A second simulation aims to 

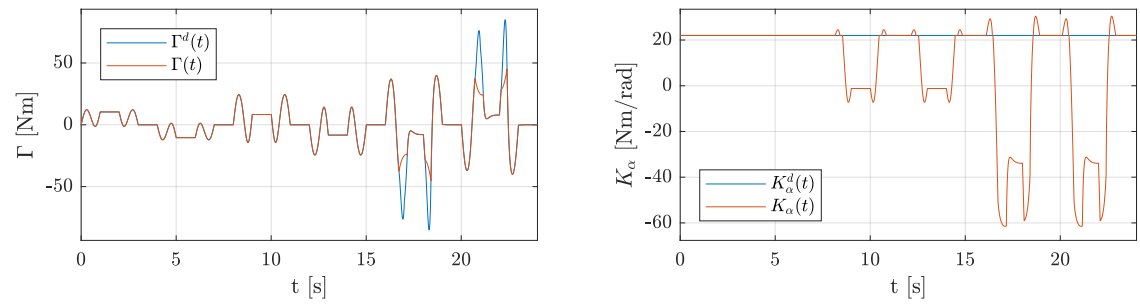

Fig. 7: Desired wrench effective wrenches $\Gamma^{d}(t), \Gamma(t)$ (left) and desired and effective stiffness $K_{\alpha}^{d}(t)=22 \mathrm{Nm} / \mathrm{rad}, K_{\alpha}(t)$ (right).

illustrate the behavior that can be obtained when the WFW is discontinuous. The parameters of the simulation are the same as previously but the spring stiffness is $k_{1}=k_{2}=50 \mathrm{~N} / \mathrm{m}$. As illustrated in Fig. 3, configurations $\pi / 4$ and $3 \pi / 4$ belong to the WFW while configuration $\pi / 2$ does not. Fig. 8 shows that:

- for a desired motion with a final configuration $\pi / 4$, the reference is nicely tracked;

- for a desired motion with a final configuration $3 \pi / 4$, the convergence toward the final configuration is obtained, the unstable configuration can be stabilized. Some tracking errors are observed during the transition phase, this can be explained by dynamical effects and by the fact that the intermediate configurations are not reachable without saturating the actuator forces. However, the mechanism can reach the second region of the WFW for a sufficiently high initial velocity;

- for a desired motion finishing at $\pi / 2$, which is outside the WFW, oscillations appear before stabilization at the limit of the WFW. Stabilization exists as damping due to friction effects was added in the system.

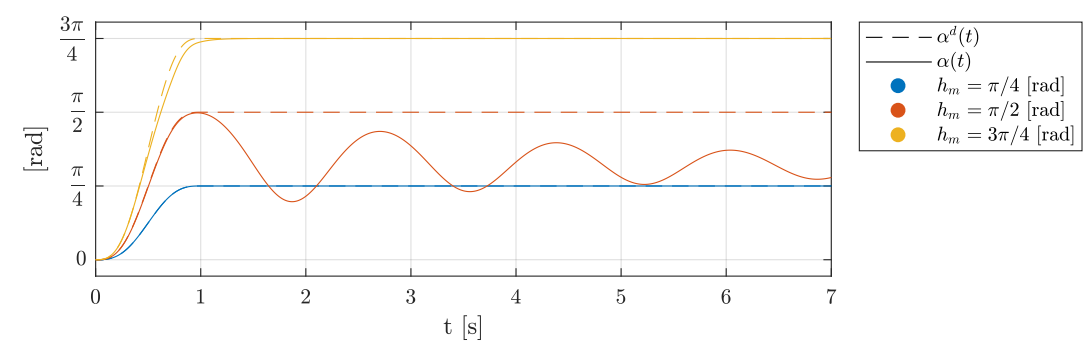

Fig. 8: Skew-sine reference angular displacement $\alpha^{d}(t)$ and simulated angular displacement $\alpha(t)$ with a discontinuous WFW $\left(k_{1}=k_{2}=50 \mathrm{~N} / \mathrm{m}\right)$.

The simulation uses a computed torque control. A simpler control law as a PID control with or without compensation of the static term can also been 
used. In this case, errors in the motion tracking will appear but in any case, the distribution of forces to choose the stiffness module can be applied.

\section{Conclusions}

An antagonistically actuated tensegrity mechanism subject to gravity was studied in this paper. Depending on the mechanism parameters, the workspace was shown to be not necessarily connected but it was possible to jump between two separated regions. Due to the actuation redundancy, both motion and stiffness could be controlled but within certain limits. The mechanism was shown to exhibit negative stiffnesses in some parts of its workspace, which can help it escape from obstacle collisions. Future work will focus on the optimal design.

Acknowledgement This work was conducted with the support of the French National Research Agency (AVINECK Project ANR-16-CE33-0025).

\section{References}

1. Skelton, R. and de Oliveira, M., Tensegrity Systems. Springer, 2009

2. M. Arsenault and C. M. Gosselin, Kinematic, static and dynamic analysis of a planar 2-dof tensegrity mechanism, Mech. and Mach. Theory, Vol. 41(9), 1072-1089, 2006

3. P. Wenger and D. Chablat, Kinetostatic Analysis and Solution Classification of a Planar Tensegrity Mechanism, proc. 7th. Int. Workshop on Comp. Kinematics, Springer, ISBN 978-3-319-60867-9, pp422-431, 2017.

4. K. Snelson, 1965, Continuous Tension, Discontinuous Compression Structures, US Patent No. 3,169,611

5. Q. Boehler et al., Definition and computation of tensegrity mechanism workspace, ASME J. of Mechanisms and Robotics, Vol 7(4), 2015

6. JB Aldrich and RE Skelton, Time-energy optimal control of hyper-actuated mechanical systems with geometric path constraints, in 44th IEEE Conference on Decision and Control, pp 8246-8253, 2005

7. S. Chen and M. Arsenault, Analytical Computation of the Actuator and Cartesian Workspace Boundaries for a Planar 2-Degree-of-Freedom Translational Tensegrity Mechanism, Journal of Mech. and Rob., Vol. 4, 2012

8. D. L Bakker et al., Design of an environmentally interactive continuum manipulator, Proc.14th World Congress in Mechanism and Machine Science, IFToMM'2015, Taipei, Taiwan, 2015

9. D. Russel et al., Mechanical Issue Inherent in Antagonistically Actuated Systems, ASME Journal of Mechanisms and Robotics, Vol. 1, 2009.

10. C. Sultan, M. Corless and R. Skelton, Linear Dynamics of Tensegrity Structures, Eng. Structures, Vol 24, pp 671-685, 2002

11. M. Gouttefarde, J.-P. Merlet and D. Daney. Wrench-Feasible Workspace of Parallel Cable-Driven Mechanisms. ICRA'07: International Conference on Robotics and Automation, Apr 2007, Roma, Italy, pp.1492-1497, 2007

12. C. Quennouelle and C. Gosselin, Stiffness matrix of Compliant Parallel Mechanisms, in Advances in Robot Kinematics: analysis and design, pp 331-341, J. Lenarcic and P. Wenger (Eds), Springer (2008) 